\title{
SUSCEPTIBILIDAD DE VARIAS LEGUMINOSAS DE COBERTURA A LOS NEMATODOS Meloidogyne incognita Y M. arenaria
}

\author{
Felipe Coto-Umañal, Robin Gómez-Gómez ${ }^{2 / *}$, Lorena Flores-Chaves ${ }^{3}$, María Isabel González-Lutz ${ }^{4}$ \\ Palabras clave: Cultivos de cobertura; abonos verdes; manejo de malezas; agricultura sostenible. \\ Keywords: Cover crops; green manures; weed management; sustainable agriculture.
}

Recibido: 13/04/2020
Aceptado: 23/06/2020

\section{RESUMEN}

Introducción. Los cultivos de cobertura aportan múltiples beneficios al agroecosistema, como lo es la disminución de poblaciones de nematodos fitoparásitos, debido a su imposibilidad de reproducirse en plantas no susceptibles. La selección de una cobertura adecuada debe considerar la ausencia de reproducción de los nematodos, ya que coberturas moderadamente susceptibles podrían comprometer ciclos de cultivo posteriores. Objetivo. Determinar la susceptibilidad de las leguminosas de cobertura Crotalaria juncea L., Crotalaria spectabilis Roth, Vigna radiata (L.) R.Wilczek, Mucuna pruriens L. var. preta y Mucuna deeringiana (Bort) Merril a los nematodos Meloidogyne incognita (Kofoid \& White) Chitwood y $M$. arenaria (Neal) Chitwood. Materiales y métodos. Se realizaron 2 experimentos simultáneos en un invernadero de la Universidad de Costa Rica, Alajuela, Costa Rica, de abril a setiembre del 2015. Las plantas de cobertura se inocularon con 3000 huevos y estados juveniles $\left(\mathrm{J}_{2}\right)$ de ambas especies de nematodos por separado.

* Autor para correspondencia. Correo electrónico: robin.gomezgomez@ucr.ac.cr

1 Universidad de Costa Rica, Estación Experimental Agrícola Fabio Baudrit Moreno, Alajuela, Costa Rica. (D) 0000-0003-1650-6869.

2 Universidad de Costa Rica, Estación Experimental Agrícola Fabio Baudrit Moreno, Alajuela, Costa Rica. (D) 0000-0002-8543-8137.
ABSTRACT

Susceptibility of legume cover crops to the nematodes Meloidogyne incognita and $M$. arenaria. Introduction. Cover crops provide multiple benefits to the agroecosystem, among them is their capacity to diminish populations of phytoparasitic nematodes due to the nematodes inhability to reproduce in non susceptible plants. The selection of an adecuate living mulch must be based on the absence of nematode reproduction, because moderately susceptible mulches could compromise future crop cycles. Objective. To determine the susceptibility of the legume cover crops Crotalaria juncea L., Crotalaria spectabilis Roth, Vigna radiata (L.) R.Wilczek, Mucuna pruriens L. var. preta, and Mucuna deeringiana (Bort) Merril to the nematodes Meloidogyne incognita (Kofoid \& White) Chitwood and $M$. arenaria (Neal) Chitwood. Materials and methods. Two experiments were carried out simultaneously in a greenhouse at the Estación Experimental Agrícola Fabio Baudrit Moreno, Universidad de Costa Rica, located in Alajuela, from April to September 2015. Legumes

3 Universidad de Costa Rica, Centro de Investigaciones en Protección de Cultivos, San José, Costa Rica. (iD) 0000-0002-2622-2707.

4 Universidad de Costa Rica, Escuela de Estadística, San José, Costa Rica. (D) 0000-0002-3073-7746. 
Se utilizó un diseño experimental completamente al azar. Resultados. No se encontraron diferencias significativas entre plantas inoculadas y sin inocular, en las variables índice de masas de huevos (IMH), factor de reproducción (FR), peso seco de la parte aérea y peso fresco de raíces. Las poblaciones de $M$. incognita y $M$. arenaria disminuyeron en promedio un $71 \%$ y $19 \%$, respectivamente, en $V$. radiata, y un $32 \%$ y $29 \%$, respectivamente, en $C$. spectablis. No se observaron masas de huevos ni agallas en las raíces de las 2 especies de Mucuna ni en C. spectabilis. Vigna radiata y $C$. juncea presentaron masas de huevos, pero no superaron la categoría 1 según la escala utilizada (0-5). Conclusión. Todas las especies de leguminosas evaluadas en este estudio disminuyeron las poblaciones de nematodos inoculados, lo cual sugiere que el establecimiento de estas leguminosas de cobertura podría ser una estrategia viable para el manejo de poblaciones de Meloidogyne en campos agrícolas.

\section{INTRODUCCIÓN}

Los cultivos de cobertura se establecen en asociación con otras plantas, ya sean intercalados o en rotación, con la finalidad de que cubran el suelo y aporten múltiples beneficios al agroecosistema. Estos cultivos, pertenecientes a múltiples familias botánicas, son especialmente importantes en las regiones tropicales con lluvias fuertes, ya que ayudan en la conservación del suelo al reducir el impacto de las gotas de lluvia, mejorar la infiltración y evitar la destrucción de la estructura del suelo y, con ello, la disminución de la escorrentía, lixiviación de nutrientes y erosión (Kaye y Quemada 2017). Al ser utilizados en sistemas de relevo o en rotación, pueden proporcionar nutrientes como nitrógeno y aportar materia orgánica al suelo, además de la supresión were inoculated with 3000 eggs and juveniles $\left(\mathrm{J}_{2}\right)$ of each nematode species, separately. A completely randomized experimental design was implemented. Results. No significant differences were determined between inoculated and noninoculated plants for the variables egg mass index (IMH), reproduction factor (FR), fresh weight of roots or dry weight of shoots. $M$. incognita and $M$. arenaria populations declined $71 \%$ and $19 \%$ in $V$. radiata plants, respectively, and $32 \%$ and $29 \%$, respectively in C. spectabilis plants. No egg masses or galls were visible in roots of both Mucuna species and C. spectabilis. Egg masses were visible in roots of $V$. radiata and $C$. juncea but were classified as category 1 according to the rating system used (0-5). Conclusion. All legume cover crops evaluated decreased nematode populations of both species inoculated, which suggests that establishment of these legume cover crops could be a viable strategy for management of Meloidogyne populations in agricultural fields.

de malezas y disminución de numerosos patógenos (Kayani et al. 2012).

Algunos cultivos de cobertura presentan el potencial de disminuir las poblaciones de nematodos fitoparásitos, ya que al no ser susceptibles a estos organismos, se disminuye o impide su multiplicación. Esta característica es deseable al diseñar estrategias de manejo complementarias al tratamiento del suelo con productos químicos (Hussain et al. 2016), además de otras prácticas culturales como la rotación de cultivos (McSorley 1998, Noe 1998, Ahmad et al. 2004, Kayani et al. 2012).

Los nematodos formadores de agallas en las raíces (Meloidogyne spp.) están distribuidos ampliamente por todo el mundo y atacan a una amplia variedad de cultivos de importancia económica (Sasser y Freckman 1987, Kinlock y Sprenkel 1994, Kamran et al. 2013). Las especies 
de Meloidogyne son especialmente importantes en las zonas tropicales y subtropicales, donde las condiciones ambientales y las prácticas de cultivo favorecen su desarrollo (Luc et al. 2005). Por su hábitat alimentario, son endoparásitos sedentarios, penetran en las raíces para alimentarse y ponen sus huevos en el exterior de la raíz (Sasser y Freckman 1987). Meloidogyne arenaria, M. incognita y $M$. javanica se encuentran ampliamente distribuidas en los trópicos, se consideran plagas clave, ya que representan el $90 \%$ de los daños causados por nematodos de este género (Castagnone-Sereno 2002).

El conocimiento de la susceptibilidad de los cultivos de cobertura a Meloidogyne spp. es crítico cuando se requiere un manejo de sus poblaciones. La gran cantidad de hospederos susceptibles a estos nematodos limita la elección de aquellos cultivos de cobertura, que no permiten su reproducción y multiplicación.

Las leguminosas de cobertura Crotalaria juncea, C. spectabilis y Vigna radiata presentan un hábito de crecimiento erecto, lo cual es deseable en sistemas de asociación con el cultivo principal, mientras que las especies de Mucuna presentan un hábito de crecimiento rastrero y trepador, característica favorable en sitios donde se requiera un periodo de descanso sin la presencia de otro cultivo. La información sobre la susceptibilidad de estas plantas a Meloidogyne spp. es escasa, por lo que el objetivo de este estudio fue determinar la susceptibilidad de las leguminosas de cobertura Crotalaria juncea L., Crotalaria spectabilis Roth, Vigna radiata (L.) R.Wilczek, Mucuna pruriens L. var. preta y Mucuna deeringiana (Bort) Merril a los nematodos Meloidogyne incognita (Kofoid \& White) Chitwood y $M$. arenaria (Neal) Chitwood.

\section{MATERIALES Y MÉTODOS}

Ubicación del estudio. El estudio se llevó a cabo de abril a setiembre del 2015, en un invernadero de la Estación Experimental Agrícola Fabio Baudrit (EEAFBM), Universidad de Costa Rica, localizado en Alajuela, Costa Rica (coordenadas $10^{\circ} 00^{\prime} 25.0^{\prime \prime} \mathrm{N}, 84^{\circ} 15^{\prime} 57.7^{\prime \prime} \mathrm{O}$ ). La precipitación promedio anual en este sitio es de $1940 \mathrm{~mm}$ distribuidos de mayo a noviembre y una temperatura promedio anual de $22^{\circ} \mathrm{C}$.

Material vegetal. Se establecieron 2 experimentos simultáneos. En el primero, se evaluaron las especies M. pruriens cv. preta (Wolf Seeds, Brasil), M. deeringiana y Vigna radiata (producidas en la EEAFBM, a partir de semilla criolla de Costa Rica). En el segundo experimento, se estudiaron $C$. juncea y $C$. spectabilis (ambas de Wolf Seeds, Brasil). Esta separación se realizó con el fin de agrupar las plantas con comportamiento y ciclo de desarrollo similar, ya que las plantas del primer experimento presentan mayor vigorosidad y desarrollo radical en comparación con las del segundo experimento. Ambos experimentos se dispusieron en un diseño completamente al azar con 4 repeticiones para cada tratamiento.

Paralelo a estos experimentos, se sembraron macetas con el cultivar de tomate Hayslip, susceptible a los nematodos, con el fin de comprobar la viabilidad infectiva del inóculo utilizado. Las plantas de tomate no formaron parte del estudio, pero fueron un referente para verificar que los nematodos tuvieran el potencial de causar agallas reproducirse y, por lo tanto, afectar el crecimiento de las plantas estudiadas.

Obtención del inóculo. Para incrementar la población de los nematodos, se preparó un almácigo con semillas de tomate puestas a germinar en bandejas con suelo previamente esterilizado con vapor de agua, según la metodología descrita por Castillo-Luna y GómezGómez (2016). Cuatro semanas después, las plántulas fueron transferidas individualmente a macetas plásticas de $3000 \mathrm{~cm}^{3}$ con suelo previamente esterilizado. Treinta días después del transplante, se realizó la inoculación, por separado, con una suspensión de huevos y juveniles en segundo estado de desarrollo $\left(\mathrm{J}_{2}\right)$ de $M$. incognita así como de $M$. arenaria, los cuales se obtuvieron a partir de poblaciones recolectadas en una 
finca comercial con siembra de tomate y fueron identificadas en el Laboratorio de Nematología de la Universidad de Costa Rica. A los 60 días después de la inoculación, el sistema radical se extrajo separadamente, de acuerdo con la especie de nematodo, cada uno se cortó en trozos de $1 \mathrm{~cm}$ y se colocó en Erlenmeyers de $500 \mathrm{ml}$, a los que se agregó $300 \mathrm{ml}$ de una solución de hipoclorito de sodio $(\mathrm{NaOCl})$ al $0,5 \%$, y se agitaron vigorosamente por 3 minutos (Barker 1985). Luego, se lavó con agua potable en cribas de 100 y 500 poros por centímetro cuadrado y se aforó la solución hasta $500 \mathrm{ml}$ con el fin de cuantificar la población total de $M$. incognita y $M$. arenaria en ese volumen. La suspensión resultante permaneció en un beaker con un inyector de burbujas de aire para su oxigenación y homogenización hasta ser utilizado en las macetas previamente sembradas con las leguminosas de cobertura.

Siembra de plantas de cobertura. Cinco semillas de cada especie de leguminosa se sembraron en potes de $4000 \mathrm{~cm}^{3}$ con suelo previamente esterilizado con vapor de agua; 10 días después se eliminaron 4 plántulas, para dejar una planta por maceta.

Inoculación de los nematodos en las leguminosas. La inoculación de cada especie de Meloidogyne se realizó a los 30 días después de la siembra (dds) de las 2 especies de Mucuna y $V$. radiata, y a los 37 dds en las 2 especies de Crotalaria, momento en el cual las plantas tenían un sistema radical lo suficientemente desarrollado para albergar las poblaciones del nematodo. Se inocularon 3000 unidades de huevos + juveniles $\left(\mathrm{J}_{2}\right)$, de cada especie de Meloidogyne, alrededor de cada planta, aproximadamente a $1 \mathrm{~cm}$ de distancia del tallo. Las plantas permanecieron en invernadero bajo riego diario. Además, dentro de ambos experimentos se mantuvieron macetas con leguminosas sin inocular para comparar su crecimiento en ausencia de nematodos.

Evaluación de poblaciones de Meloidogyne spp. en raíz y suelo. Por sus diferentes hábitos de crecimiento, las leguminosas fueron cortadas para su evaluación final a los 45 (V. radiata), 56 (Mucuna spp.) y 69 (Crotalaria spp.) días después de la inoculación de los nematodos. La escogencia de diferentes momentos de corta, se realizó para simular el período de vida que presentan estos cultivos en condiciones de campo.

El sistema radical de cada planta fue analizado en el laboratorio para determinar las poblaciones de nematodos, y se siguió la metodología descrita anteriormente para la obtención del inóculo. También, se procesaron $100 \mathrm{~cm}^{3}$ de suelo de cada maceta, mediante el método de centrifugación en solución azucarada (Caveness y Jensen 1955) y se cuantificaron las unidades de inóculo (u.i.) mediante un microscopio invertido. El valor obtenido se extrapoló al volumen total de suelo en cada maceta $\left(4000 \mathrm{~cm}^{3}\right)$ y se determinó la población final (Pf) del nematodo para cada repetición. El valor de la población final de cada especie de Meloidogyne se obtuvo al sumar $\mathrm{Pf}_{\text {suelo }}+\mathrm{Pf}_{\text {raíces }}$.

Se determinó el peso fresco de las raíces de cada una de las plantas evaluadas inmediatamente después del corte. La parte aérea de cada planta se secó en una estufa a $65^{\circ} \mathrm{C}$ durante 48 horas y se determinó el peso seco. No se realizó la medición del peso seco radical por la necesidad de procesar y, por lo tanto, destruir las raíces para la cuantificación de otras variables.

Estimación del índice de masas de huevos y factor de reproducción. Se calculó el índice de masas de huevos (IMH) modificando la escala de Taylor y Sasser (1978) que se muestra en la Tabla 1. Este análisis consistió en el conteo de las masas de huevos producidas por las hembras de Meloidogyne. Para tal fin, se lavaron y sumergieron las raíces de cada una de las plantas en una solución de Floxina B 0,05\% por 30 min para facilitar la observación y conteo de las masas de huevos. 
Tabla 1. Escala del índice de masas de huevos (IMH) del género Meloidogyne utilizada para evaluar la susceptibilidad de leguminosas de cobertura a este género de nematodos. Alajuela, Costa Rica, 2015.

\begin{tabular}{cc}
\hline $\begin{array}{c}\text { Número de masas } \\
\text { de huevos }\end{array}$ & $\begin{array}{c}\text { Índice de masas } \\
\text { de huevos (IMH) }\end{array}$ \\
\hline 0 & 0 \\
$1-2$ & 1 \\
$3-10$ & 2 \\
$11-30$ & 3 \\
$30-100$ & 4 \\
$100-200$ & 5 \\
\hline
\end{tabular}

También se determinó el factor de reproducción (FR), que establece la cantidad de veces que se reprodujo la población inicial en cada hospedero. El cálculo de esta variable se realizó mediante la fórmula:

$$
\mathrm{FR}=\mathrm{Pf} / \mathrm{Pi}
$$

Donde:

Pf $=$ población final.

$\mathrm{Pi}=$ población inicial de los nematodos inoculados.

Si $\mathrm{FR}=0$, la planta hospedera es considerada como resistente, si $\mathrm{FR}<1$ la planta es considerada como moderadamente resistente y si FR $>1$ se considera un hospedero susceptible al nematodo.
Análisis de datos. En los 2 experimentos se realizó un análisis de varianza para un diseño irrestricto aleatorio con un arreglo factorial de los tratamientos especie vegetal $\mathrm{x}$ especie nematodo. La variable índice de masas de huevos (IMH) se analizó mediante tablas de contingencia en las que se relacionaron los valores de la escala de referencia con las especies de cobertura. A causa de la presencia de muchos valores 0 , no se utilizó la prueba Chi cuadrado para determinar posibles diferencias significativas, ya que, en esas condiciones, la prueba resultaría no válida. Los valores obtenidos al calcular el factor de reproducción (FR) no fueron analizados estadísticamente, porque casi todos fueron 0 , en cuyo caso cualquier prueba estadística daría no significativa. Las variables peso fresco de raíces y peso seco de la parte aérea de las leguminosas fueron sometidas a un análisis de varianza.

\section{RESULTADOS}

No se encontraron diferencias significativas $(p=0,8769)$ en el peso seco de la parte aérea de las leguminosas al comparar las plantas inoculadas y las no inoculadas para todos los cultivos de cobertura (Tabla 2). Debido al ataque de un hongo y de una plaga vertebrada dentro del invernadero, donde se encontraban las plantas de $V$. radiata, se perdió la parte aérea de estas plantas días antes de su corta, por lo que no fue posible incluir esta especie en el análsis de esta variable.

Tabla 2. Peso seco promedio ( $\mathrm{g}$ ) de la parte aérea de 4 leguminosas inoculadas y sin inocular con M. incognita y M. arenaria. Alajuela, Costa Rica, 2015.

\begin{tabular}{lccc}
\hline Cultivo de cobertura & M. incognita & M. arenaria & Sin inocular \\
\hline Experimento 1 & 19,97 & & \\
Mucuna pruriens & 26,84 & 12,35 & 16,78 \\
Mucuna deeringiana & & 39,46 & 24,88 \\
Experimento 2 & 15,10 & & \\
Crotalaria juncea & 24,50 & 15,49 & 12,91 \\
Crotalaria spectabilis & & 30,01 & 35,45 \\
\hline
\end{tabular}


De manera similar, tampoco se presentaron diferencias significativas $(p=0,5665)$ en el peso fresco de las raíces de las leguminosas entre aquellas plantas inoculadas con los nematodos y las no inoculadas (Tabla 3).

Tabla 3. Peso fresco promedio (g) de las raíces de 5 leguminosas inoculadas y sin inocular con los nematodos M. incognita o M. arenaria. Alajuela, Costa Rica, 2015.

\begin{tabular}{lccc}
\hline Cultivo de cobertura & M. incognita & M. arenaria & Sin inocular \\
\hline Experimento 1 & & & \\
Mucuna pruriens & 74,08 & 64,90 & 97,90 \\
Mucuna deeringiana & 123,28 & 123,10 & 151,53 \\
Vigna radiata & 2,60 & 3,73 & 3,90 \\
Experimento 2 & & & \\
Crotalaria juncea & 20,43 & 20,23 & 14,40 \\
Crotalaria spectabilis & 37,75 & 25,53 & 31,08 \\
\hline
\end{tabular}

$M$. incognita y $M$. arenaria solo se reprodujeron en las raíces de las leguminosas $V$. radiata y C. spectabilis (Tabla 4). Sin embargo, los valores promedio del FR fueron inferiores a 1, lo que provocó que la población inicialmente inoculada de $M$. incognita y $M$. arenaria disminuyera en promedio un $71 \%$ y $19 \%$, respectivamente, en V. radiata, y un $32 \%$ y $29 \%$ en C. spectablis. En comparación, en las plantas de tomate inoculadas con los nematodos para comprobar la infectividad del inóculo utilizado, el FR de $M$. incognita fue de 161 y el FR de $M$. arenaria fue de 66 .

Tabla 4. Factor de Reproducción (FR) promedio de los nematodos Meloidogyne incognita y M. arenaria en el sistema raízsuelo de 5 leguminosas de cobertura. Alajuela, Costa Rica, 2015.

\begin{tabular}{lcc}
\hline Cultivo de cobertura & M. incognita & M. arenaria \\
\hline Experimento 1 & 0 & \\
Mucuna pruriens & 0 & 0 \\
Mucuna deeringiana & 0,3 & 0 \\
Vigna radiata & & 0,8 \\
Experimento 2 & 0 & 0 \\
Crotalaria juncea & 0,7 & 0,7 \\
Crotalaria spectabilis & & \\
\hline
\end{tabular}

Ninguna de las 2 especies de nematodo se lograron reproducir $(\mathrm{FR}=0)$ en las leguminosas C. juncea y Mucuna spp., por lo cual estas leguminosas son consideradas como resistentes (Oostenbrink 1966).

No se observaron masas de huevos ni agallas de las 2 especies de Meloidogyne en las raíces de Mucuna y C. spectabilis (Tabla 5). Solo las plantas de $V$. radiata y $C$. juncea tenían masas de huevos, pero no superaron la categoría 1, según la escala utilizada. Las plantas de tomate indicadoras fueron clasificadas con un IMH de 5, la máxima categoría de esta escala para ambos nematodos estudiados. 
Tabla 5. Índice de masas de huevos promedio observados en el sistema radical de 5 leguminosas de cobertura inoculadas con M. incognita y M. arenaria. Alajuela, Costa Rica, 2015.

\begin{tabular}{lcc}
\hline Cultivo de cobertura & M. incognita & M. arenaria \\
\hline Experimento 1 & 0 & 0 \\
Mucuna pruriens & 0 & 0 \\
Mucuna deeringiana & 1 & 1 \\
Vigna radiata & & \\
Experimento 2 & 1 & 0 \\
Crotalaria juncea & 0 & 0 \\
Crotalaria spectabilis & & \\
\hline
\end{tabular}

\section{DISCUSIÓN}

Los resultados obtenidos en este estudio mostraron que las leguminosas $M$. pruriens, $M$. deeringiana, V. radiata, C. juncea y C. spectabilis no son susceptibles a los nematodos $M$. incognita y $M$. arenaria; consecuentemente, se presentó una disminución en las poblaciones de ambas especies de fitoparásitos.

Las especies de nematodos inoculadas no afectaron el peso de raíz de las coberturas, lo cual podría estar ligado a factores de resistencia, según Sañudo y Betancourth (2005). Esas personas autoras, indicaron que en materiales resistentes y moderadamente resistentes se dificulta el proceso de infección en estados como pre-penetración, penetración y reproducción, ya que impiden el acoplamiento en las células radicales y posteriormente, en los sitios de alimentación, lo que reduce significativamente la tasa de reproducción del nematodo. Al no verse alterados negativamente procesos clave como el transporte de agua y asimilados, no se afecta en gran medida el correcto funcionamiento fisiológico de la planta. En este sentido, componentes de su crecimiento, como el peso radical, continúan su desarrollo normal y no se afectan negativamente, tal y como se observó en todas las coberturas con y sin inóculo.

En comparación, las plantas de tomate utilizadas como indicadores presentaron marchitez generalizada en las horas más calurosas del día, esto posiblemente debido a este daño en los sistemas de transporte de sustancias. Se observaron en esas plantas síntomas propios de la deficiencia de agua y nutrientes, aun cuando estos factores estuvieron regulados durante el transcurso del experimento. También se observó formación de agallas en las raíces.

La baja producción de agallas, huevos y en particular el bajo factor de reproducción, sugieren que existen mecanismos de resistencia presentes en las plantas de cobertura. Sikder y Vestergard (2020) mencionan la producción de alcaloides, metabolitos secundarios y sustancias aleloquímicas como componentes de tales mecanismos. En ese sentido, se ha reportado que Mucuna pruriens y Vigna unguiculata presentan alguna capacidad de resistencia en la etapa de preinfección, en la cual liberan sustancias en forma de exudados radicales, los cuales se mantienen en la rizosfera y evitan la penetración de juveniles antes de que logren la infección (Pofu y Mashela 2011). Por su parte, la presencia de los alcaloides monocrotalina y pyrrolizidina, presentes en especies de Crotalaria pueden afectar o inhibir el movimiento de juveniles de $M$. incognita (Chitwood 2002). Aun así, la presencia de estos mecanismos de resistencia deberá ser confirmada y estudiada con mayor detalle en investigaciones futuras.

Los resultados obtenidos también fueron consistentes con otros estudios en los que se utilizaron plantas de cobertura como Ricinus communis, Aeschynomene americana (Rodríguez-Kabana et al. 1992), Mucuna spp. 
(McSorley y Gallaher 1992), C. spectabilis (Good et al. 1965), Indigofera hirsuta (Rhoades 1964), para el manejo de M. arenaria y otras especies de Meloidogyne. Por su parte, Claudius-Cole et al. (2014) evaluaron el potencial de 10 cultivos de cobertura, entre ellos $C$. juncea y M. pruriens, y determinaron que dichas leguminosas fueron efectivas en reducir significativamente las poblaciones de Meloidogyne spp., comparado con un ciclo previo en el que no se utilizaron leguminosas de cobertura.

La selección de un cultivo de cobertura adecuado para el control de poblaciones de nematodos debe enfocarse en la ausencia de reproducción por parte de estos, ya que cultivos con una categoría de susceptibilidad moderada podrían comprometer ciclos de cultivo posteriores, sobre todo si estos carecen de algún grado de resistencia a estas plagas. Sin embargo, se ha reportado el caso de cultivos de cobertura que actúan como trampa para nematodos endoparásitos, de manera que los huevos dan lugar a juveniles en presencia de la cobertura, pero después los juveniles mueren de inanición, ya que no se pueden alimentar, como consecuencia de esto se reducen significativamente las poblaciones en el campo (Siddiqui y Alam 1988, Evenhuis et al. 2004, Pudasaini et al. 2006). Además se debe prestar especial atención en la selección de la leguminosa, ya que un cultivo de cobertura exitoso en reducir la densidad de uno o varios géneros de nematodo, puede que no tenga efecto con otras especies de nematodos y/o sus diferentes razas. Así, por ejemplo, Claudius-Cole et al. (2014) concluyeron que Crotalaria fue efectivo contra Meloidogyne, pero al mismo tiempo incrementó las poblaciones de los nematodos Pratylenchus y Scutellonema.

En conclusión, M. pruriens, M. deeringiana, $V$. radiata, C. juncea y $C$. spectabilis no presentaron susceptibilidad a $M$. incognita y $M$. arenaria, por lo que consecuentemente, se presentó una disminución en las poblaciones del nematodo. No se observaron diferencias en el crecimiento, determinado por el peso aéreo y de raíces, de las leguminosas de cobertura inoculadas con los nematodos y aquellas no inoculadas.

\section{LITERATURA CITADA}

Ahmad, M; Mukhtar, T; Ahmad, R. 2004. Some studies on the control of citrus nematode (Tylenchulus semipenetrans) by leaf extracts of three plants and their effects on plant growth variables. Asian Journal of Plant Science 3:544-548. Doi:10.3923/ ajps.2004.544.548.

Barker, KR. 1985. Nematode extraction and bioassays. In Barker, KR; Carter, CC; Sasser, JN (eds.). An Advanced Treatise on Meloidogyne. Vol. II: Methodology. North Carolina State University, Raleigh, USA. p 18-35.

Castagnone-Sereno, P. 2002. Genetic variability in parthenogenetic root-knot nematodes, Meloidogyne spp. and their ability to overcome plant resistance genes. Nematology 4:605-608. Doi:10.1163/15685410260438872.

Castillo-Luna, MP; Gómez-Gómez, R. 2016. Efecto de la esterilización del suelo con vapor de agua sobre semillas de malezas. Agronomía Mesoamericana 27:409-413. Doi:10.15517/am.v27i2.21286.

Caveness, F; Jensen, H. 1955. Modification of the centrifugal flotation technique for the isolation and concentration of nematodes and their eggs from soil plant tissue. Proceedings of the Helminthological Society 22(2):87-89.

Chitwood, DJ. 2002. Phytochemical based strategies for nematode control. Annual Review of Phytopathology 40:221-222. Doi:10.1146/annurev. phyto.40.032602.130045.

Claudius-Cole, AO; Fawole, B; Asiedu, R; Coyne, DL. 2014. Management of Meloidogyne incognita in yam-based cropping systems with cover crops. Crop Protection 63:97-102. Doi:10.1016/j.cropro.2014.05.011.

Evenhuis, A; Korthals, GW; Molendijk, LPG. 2004. Tagetes patula as an effective catch crop for long-term control of Pratylenchus penetrans. Nematology 6:877-881. Doi:10.11163/15685410440338632.

Good, J; Minton, N; Jaworski, C. 1965. Relative susceptibility of selected cover crops and coastal bermudagrass to plant nematodes. Phytopathology 55:1026-1030.

Hussain, M; Kamran, M; Singh, K; Zouhar, M; Rysanek, P; Anwar, SA. 2016. Response of selected okra cultivars to Meloidogyne incognita. Crop Protection 82:1-6. Doi:10.1016/j.cropro.2015.12.024.

Kamran, M; Anwar, SA; Javed, N; Khan, SA; Abbas, H; Iqbal, MA; Zohaib, A. 2013. The influence of Meloidogyne incognita density on susceptible tomato. Pakistan Journal of Zoology 45:727-732.

Kayani, MZ; Mukhtar, T; Hussain, MA. 2012. Evaluation of nematicidal effects of Cannabis sativa L. and Zanthoxylum alatum Roxb. against root-knot nematodes Meloidogyne incognita. Crop Protection 39:52-56. Doi: 10.1016/j.cropro.2012.04.005. 
Kaye, JP; Quemada, M. 2017. Using cover crops to mitigate and adapt to climate change. A review. Agronomy for Sustainable Development 37(1):4. Doi: https:// doi.org/10.1007/s13593-016-0410-x

Kinlock, RA; Sprenkel, RK. 1994. Plant-parasitic nematodes associated with cotton in Florida. Journal of Nematology 26:749-752.

Luc, M; Sikora, RA; Bridge, J. 2005. Plant parasitic nematodes in subtropical and tropical agriculture. $2^{\mathrm{a}}$ edición. CABI Publishing, Egham, Reino Unido. $831 \mathrm{p}$.

McSorley, R. 1998. Alternative practices for managing plant-parasitic nematodes. Journal of Alternative Agriculture 13:98-104. Doi:10.1017/ S0889189300007761.

McSorley, R; Gallaher, RN. 1992. Comparison of nematode population densities on six summer crops at seven sites in north Florida. Journal of Nematology 24:699-706.

Noe, JP. 1998. Crop and nematode-management systems. In Barker, KR; Pederson, GA; Windham, GL; Bartels, JM (eds.). Plant and nematode interactions. Agronomy Monograph №. 36. ASA, CSSA, and SSSA, Madison, WI, USA. p. 159-171.DOI: https:// doi.org/10.2134/agronmonogr36.c8

Oostenbrink, R. 1966. Major characteristics of the relation between nematodes and plants. Mededelingen Landbouwhogeschool Wageningen 66:1-46.

Pofu, KM; Mashela, PW. 2011. Using relative penetration and maleness indices in Meloidogyne incognita to establish resistance type in Cucumis myriocarpus. African Journal of Biotechnology 10:390-393. Doi: 10.5897/AJB10.1584.
Pudasaini, MP; Viaene, N; Moens, M. 2006. Effect of marigold (Tagetes patula) on population dynamics of Pratylenchus penetrans in a field. Journal of Nematology 8:477-484. Doi: 10.1163/156854106778613930.

Rhoades, H. 1964. Effect of Crotalaria spectabilis and Sesbania exaltata on plant nematode populations and subsequent yiel of snap beans and cabbage. Florida State Horticulture Society 77:233-237.

Rodríguez-Kabana, R; Pinochet, J; Robertson, DG; Wells, L. 1992. Crop rotation studies with velvetbean (Mucuna deeringiana) for the management of Meloidogyne spp. Nematropica 22:45-53.

Sasser, J; Freckman, D. 1987. A world perspective on nematology: the role of society. In Veech, JA; Dickson, DW (eds.). Vistas on nematology: A commemoration of the twenty-fifth anniversary of the society of nematologist. Society of Nematologist, Madison, WI, USA. p. 7-14.

Siddiqui, MA; Alam, MM. 1988. Studies on the nematotoxicity of root exudates of certain species of Tagetes. Indian Journal of Nematology 18:335-337.

Sikder, MM; Vestergård, M. 2020. Impacts of root metabolites on soil nematodes. Frontiers in plant science 10:1792. DOI: https://doi.org/10.3389/ fpls.2019.01792

Sañudo, B; Betancourth, C. 2005. Fundamentos de fitomejoramiento. ed. Universidad de Nariño, Pasto, Colombia. $150 \mathrm{p}$.

Taylor, A; Sasser, J. 1978. Biology, identification and control of root-knot nematodes (Meloidogyne species). Department of Plant Pathology, North Carolina State University, NC, USA. 111 p. 
